\title{
Potent Anticancer Effect of PET-Fraction (PET-F) in Comparison with Other Commercial Products on Canine Cancer Cells
}

\author{
Sensuke Konno*, Muhammad Choudhury \\ Department of Urology, New York Medical College, Valhalla, USA \\ Email: sensuke_konno@nymc.edu
}

Received 1 April 2015; accepted 15 May 2015; published 18 May 2015

Copyright (C) 2015 by authors and Scientific Research Publishing Inc. This work is licensed under the Creative Commons Attribution International License (CC BY). http://creativecommons.org/licenses/by/4.0/

(c) (i) Open Access

\begin{abstract}
A variety of nutritional supplements are commercially available for the potential therapeutic purpose for dogs and other domestic animals with cancers. However, the efficacy of these supplements on those cancer-bearing animals has not been verified and remains uncertain, due to the lack of the sufficient scientific studies/data. To address this important issue, we examined actual anticancer effects of five selected commercial products on the two types of canine cancer models in vitro. Among them, PET-Fraction (PET-F) obtained from maitake mushroom has been shown to have potent anticancer effect on canine cancer cells. In comparison with this PET-F, possible anticancer effects of the rest of four products were examined. Two of four products were found to have the significant anticancer effects on both cancer cells, while other two products had little effects. Nevertheless, PET-F exhibited the best anticancer effect. Cell cycle analysis indicated that the PET$F$-induced growth reduction was attributed to a $G_{1}$ cell cycle arrest, and Western blot analysis further revealed that such a growth reduction was coupled with apoptosis. Moreover, anticancer activity of PET-F was remarkably enhanced with vitamin C, but no such synergistic effect was seen with other four products. Therefore, although two other products besides PET-F yet have anticancer activities, PET-F appears to be the most potent and promising commercial product that can be useful in effectively and safely treating canine cancers.
\end{abstract}

\section{Keywords}

Canine Cancer Cells, Commercial Supplements, PET-Fraction, Vitamin C, Anticancer

\footnotetext{
${ }^{*}$ Corresponding author.
}

How to cite this paper: Konno, S. and Choudhury, M. (2015) Potent Anticancer Effect of PET-Fraction (PET-F) in Comparison with Other Commercial Products on Canine Cancer Cells. Open Journal of Veterinary Medicine, 5, 101-110. 


\section{Introduction}

The dog is often known as a best and close companion of man and is exposed to the same environmental factors/conditions as we are [1]. That can be at least one of the reasons why dogs are also prone to various types of cancers found in humans. Cancer is indeed the leading cause of death in dogs over 10 years and one in four dogs will die from it [2]. Although several therapeutic options are available, unfortunately it is the fact that the effective or satisfactory modalities have not yet been established. Hence, it is urgently required for finding the more effective treatments. To explore the better way to treat such canine cancers, we have been pursuing an alternative approach using natural agents or products with anticancer activities.

Today, a variety of commercial products are available as nutritional supplements for general health maintenance or therapeutic purpose. However, due to the lack of the sufficient scientific studies/data, the actual efficacy of these supplements on dogs and other domestic animals bearing cancers needs to be adequately addressed. It is also challenging to properly assess such an efficacy of many "unverified" supplements on those animals with cancers. Among them, we are especially interested in "PET-Fraction" (PET-F)" extracted from maitake mushroom (Grifola frondosa) that is an edible, tasty, and medicinal mushroom [3]. Originally, PET-F was developed as a nutritional supplement called, "D-fraction (DF)", for human use. Characteristically, DF is the proteinbound polysaccharide known as $\beta$-glucan with a molecular weight of $\sim 1 \times 10^{6}$ dalton [3] [4]. Unlike many of those unverified commercial products, DF has been extensively and scientifically studied over 30 years, revealing numerous physiological benefits such as immunomodulatory, antitumor/anticancer, and antiviral activities [4]-[11]. Following these encouraging findings, DF has been specifically developed for veterinary use known as "DVM Fraction TM" [12], which is further improved and named as PET-Fraction"T (PET-F). DVM Fraction indeed has demonstrated a potent anticancer effect on canine cancer cells in vitro [12]; however, an increasing number of commercial products (for a therapeutic purpose) have been introduced to the marketplace without having been tested or approved for their actual efficacy. Hence, our aim was to compare some of commercial products with the current PET-F in terms of anticancer effect on canine cancer cells. We selected four commercial products, which were specific mushroom extracts or having active glucan components, so that they were relevant to PET-F (a mushroom extract with $\beta$-glucan) for comparison. The followings are chosen for this study, but the commercial names of some products are unrevealed, or rather, they are described by the types of mushrooms or glucans: 1) AG, a bioactive extract from agaricus mushroom; 2) HM, a bioactive extract from hime-matsutake (Iwade 101); 3) AHCC, an $\alpha$-glucan rich compound obtained from the mycelia of shiitake mushroom; 4) YBG, a $\beta$-glucan isolated from yeast cell wall. It should be noted that the actual products sold in the stores are used, but detailed ingredients or purity of these products is not described here because our intention is to assess any anticancer effects of actually sold products.

Accordingly, possible anticancer effects of five products (including PET-F) were examined on canine cancer cells in vitro. To have a better understanding of their anticancer effects, the regulatory effect on the cell cycle and induction of apoptosis were also examined by cell cycle analysis and Western blot analysis, respectively.

\section{Materials and Methods}

\subsection{Cell Culture}

The two types of canine cancer cell lines, mammary gland cancer (CF33) and soft tissue cancer (CF21), were obtained from the American Type Culture Collection (ATCC; Manassas, VA) and employed in this study. Both cells were cultured in Dulbecco's modified Eagle's (DME) medium, supplemented with 10\% fetal bovine serum, penicillin $(100 \mathrm{U} / \mathrm{ml})$, and streptomycin $(100 \mu \mathrm{g} / \mathrm{ml})$. They were maintained at $37^{\circ} \mathrm{C}$ in a humidified incubator in an atmosphere of $95 \%$ air and $5 \% \mathrm{CO}_{2}$. The medium was routinely changed every 3 days and cells were passaged weekly. For experiments, all five commercial products, AG, HM, AHCC, YBG, and PET-F, were tested for their anticancer activities in CF33 and CF21 cells. They were seeded at the initial cell density of $1 \times 10^{5}$ cells $/ \mathrm{ml}$ in the 6-well culture plates and cultured with varying concentrations $(0,200,500,700$ and $1000 \mu \mathrm{g} / \mathrm{ml})$ of five commercial products. After $72 \mathrm{~h}$, cell growth/viability was assessed by MTT assay described below.

\subsection{MTT Assay}

Cell growth/viability was determined by MTT (3-[4,5-dimethylthiazol-2-yl]-2,5-diphenyl-tetrazolium bromide) assay following the vendor's protocol (Sigma-Aldrich, St. Louis, MO). This assay is based on the conversion of 
the water-soluble MTT to an insoluble purple formazan through mitochondrial dehydrogenases in viable cells. Briefly, at the harvest time, $1 \mathrm{ml}$ of MTT reagent $(1 \mathrm{mg} / \mathrm{ml})$ prepared in phosphate-buffered saline (PBS) was added to each well of the 6-well plate, which was then incubated at $37^{\circ} \mathrm{C}$ for $3 \mathrm{~h}$. After removing MTT, DMSO (dimethyl sulfoxide) was added to each well to dissolve the formazan crystals (with purple color). Absorbance of formazan solution was read on a microplate reader, and cell growth was expressed by the percent (\%) of viable cells relative to the control reading $(100 \%)$.

\subsection{Cell Cycle Analysis}

Cell cycle phase distributions were determined on a FACScan flow cytometer (Becton-Dickinson, San Jose, CA) equipped with a double discrimination module. Control or agents-treated cells $\left(\sim 1 \times 10^{6}\right.$ cells per condition) were resuspended in $500 \mu \mathrm{l}$ of propidium iodide solution $(20 \mu \mathrm{g} / \mathrm{ml}$ propidium iodide, $0.2 \mathrm{mg} / \mathrm{ml}$ RNase, 0.2 $\mathrm{mg} / \mathrm{ml}$ EDTA, $0.5 \%$ Nonidet P-40) and incubated for $1 \mathrm{~h}$ at room temperature in the dark. Approximately 10,000 nuclei from each sample were analyzed on a flow cytometer, and CellFit software was used to quantify cell cycle compartments to estimate the $\%$ of cells distributed in the different cell cycle phases.

\subsection{Western Blot Analysis}

The procedures essentially followed the protocol described elsewhere [13]. Cell lysates were first obtained by freezing cells in liquid nitrogen, immediately followed by thawing them in a $40^{\circ} \mathrm{C}$ water bath. After this "freeze-thaw" was repeated three times, cell lysates were obtained by centrifugation at $15,000 \mathrm{rpm}$ for $10 \mathrm{~min}$ at $4^{\circ} \mathrm{C}$. An equal amount of cell lysates $(7 \mu \mathrm{g})$ was subjected to $10 \%$ SDS-polyacrylamide gel electrophoresis and transferred to a nitrocellulose membrane. The blot (membrane) was incubated for $90 \mathrm{~min}$ with the primary antibodies against bcl-2 or Bax (Santa Cruz Biotechnology, Santa Cruz, CA), followed by 30-min incubation with the appropriate secondary antibody conjugates. The specific immunoreactive protein bands (bcl-2 or Bax) were then detected by chemiluminescence following the manufacturer's protocol (Kirkegaard and Perry Laboratories, Gaithersberg, MD).

\subsection{Statistical Analysis}

All data are presented as mean \pm SD (standard deviation), and statistical differences between groups were assessed with either one-way analysis of variance (ANOVA) or the unpaired Student's $t$ test. Values of $p<0.05$ were considered to indicate statistical significance.

\section{Results}

\subsection{Anticancer Effects of Commercial Products on Canine Cancer Cells}

Both CF33 and CF21 cells were treated with five different commercial products, AG, HM, AHCC, YBG, and PET-F, at varying concentrations $(0,200,500,700$ and $1000 \mu \mathrm{g} / \mathrm{ml})$ for $72 \mathrm{~h}$. Cell growth was then determined by MTT assay because anticancer effects of these products can be assessed by the reduction/inhibition (\%) in cell growth relative to controls (100\%). For CF33 cells (Figure 1(a)), PET-F $\geq 500 \mu \mathrm{g} / \mathrm{ml}$ induced a maximum of $\sim 47 \%$ growth reduction while $\mathrm{AG}$ and $\mathrm{HM} \geq 700 \mu \mathrm{g} / \mathrm{ml}$ led to only a $\sim 20 \%$ and $\sim 26 \%$ maximum growth reduction, respectively. However, no such effects were seen by AHCC and YBG even at the highest concentration $(1000 \mu \mathrm{g} / \mathrm{ml}$ ). For CF21 cells (Figure 1(b)), both HM and PET-F $\geq 500 \mu \mathrm{g} / \mathrm{ml}$ led to a maximum growth reduction of $\sim 32 \%$ and $\sim 51 \%$, respectively, while $\mathrm{AG} \geq 700 \mu \mathrm{g} / \mathrm{ml}$ resulted in only a $\sim 27 \%$ growth reduction. AHCC and YBG yet showed no effects. Thus, only three products, AG, HM, and PET-F, were found to have anticancer activities but PET-F had the most potent anticancer effect on both cancer cells.

\subsection{Effects on Cell Cycle}

To explore the antiproliferative or anticancer mechanism(s) of these products, their possible effects on cell cycle were examined. Both CF33 and CF21 cells were treated with AG, HM, or PET-F at the effective concentration of $700 \mu \mathrm{g} / \mathrm{ml}$, whereas both AHCC and YBG were temporarily put aside due to their ineffectiveness (Figure 1). Following 3-day treatment with these products, cells were subjected to cell cycle analysis. The results showed that a statistically significant decrease in the S-phase cell number (\%) with a concomitant significant increase in 


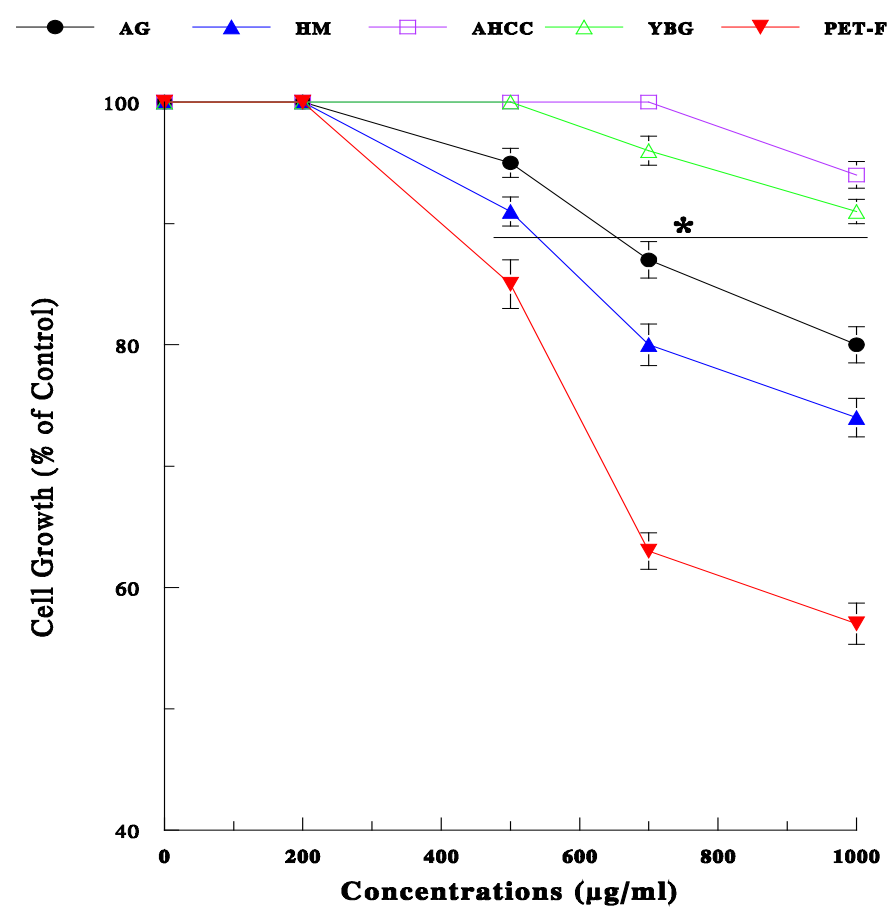

(a)

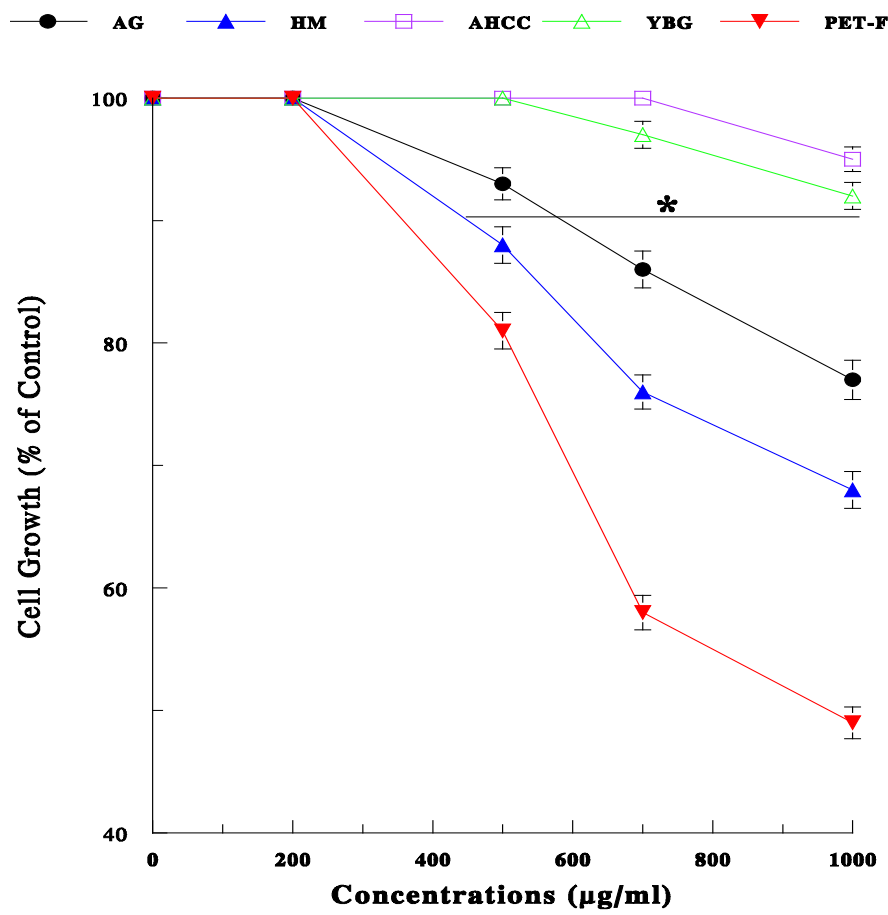

(b)

Figure 1. Effects of commercial products on the growth of two canine cancer cells. CF33 (a) and CF 21 (b) cells were treated with five different products, AG, HM, AHCC, YBG, and PET-F, at varying concentrations $(0-1000 \mu \mathrm{g} / \mathrm{ml})$. Cell growth was determined by MTT assay in $72 \mathrm{~h}$ to assess anticancer effects of these products and expressed by the percent (\%) of viable cell number relative to controls $(100 \%)$. All data were calculated as mean \pm SD (standard deviation) from three separate experiments. The data points below the line with an asterisk in the panels (a) and (b) are statistically different $(p<0.05$ compared with controls). 
the $\mathrm{G}_{1}$-phase cell number (\%) was found in both CF33 and CF22 cells with PET-F treatment (Figure 2(a) and Figure 2(b)), indicating an accumulation of cells in the $\mathrm{G}_{1}$ phase known as a $\mathrm{G}_{1}$ cell cycle arrest [14]. However, any significant change in cell cycle phases was not attained with AG or HM (Figure 2(a) and Figure 2(b)). Thus, these results suggest that the significant growth reduction induced by PET-F in CF33 and CF21 cells is more likely attributed to a $\mathrm{G}_{1}$ cell cycle arrest while little effects on cell cycle are seen with $\mathrm{AG}$ and $\mathrm{HM}$.

\subsection{Induction of Apoptosis by PET-F}

It was crucial to also examine if a $\mathrm{G}_{1}$ cell cycle arrest would ultimately lead to apoptosis, accounting for the resulting growth reduction. Both CF33 and CF21 cells were treated with AG, HM, or PET-F at the highest concentration of $1000 \mu \mathrm{g} / \mathrm{ml}$ for $24 \mathrm{~h}$, and cells were subjected to Western blot analysis on two key apoptotic regulators, bcl-2 and Bax [15]. Figure 3(a) shows autoradiographs of bcl-2 and Bax protein bands detected in CF33 cells treated with three commercial products. The expression or intensity of bcl-2 band with PET-F treatment was drastically reduced or diminished, whereas little effects on bcl-2 bands were seen with AG or HM, remaining the same levels as controls. On the other hand, a Bax band was highly expressed or enhanced with PET-F but it remained at the basal levels with AG or HM similar to controls (Figure 3(a)). The same analysis was also performed in CF21 cells and the results (autoradiographs) are shown in Figure 3(b). Like CF33 cells, CF21

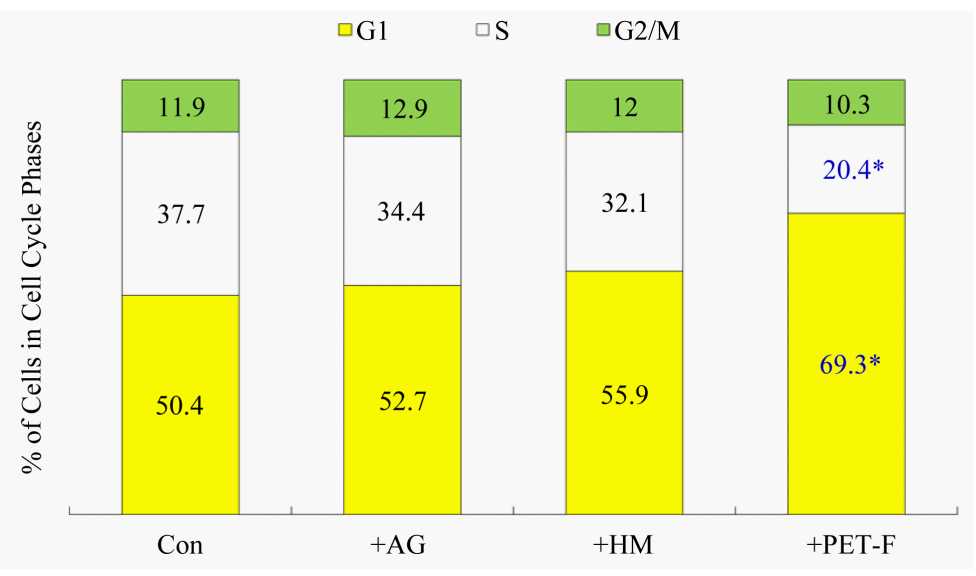

(a)

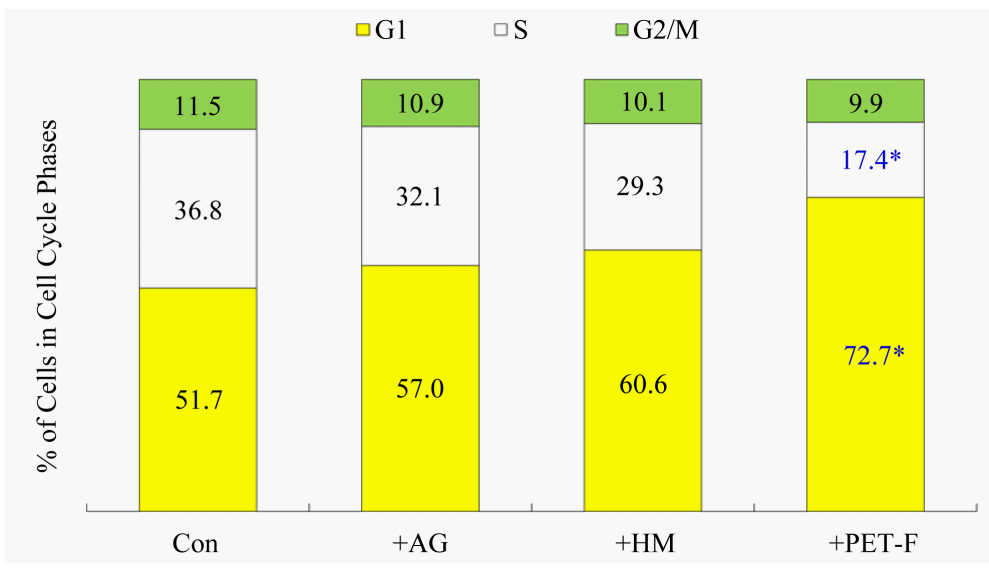

(b)

Figure 2. Cell cycle analysis. Both CF33 (a) and CF21 (b) cells were treated with AG, HM, or PET-F at the effective concentration of $700 \mu \mathrm{g} / \mathrm{ml}$. After 72 $\mathrm{h}$, both cancer cells were subjected to cell cycle analysis and the $\%$ of cell distribution in each cell cycle phase $\left(\mathrm{G}_{1}, \mathrm{~S}\right.$, and $\left.\mathrm{G}_{2} / \mathrm{M}\right)$ is plotted in the graphs. All data are mean \pm SD from three independent experiments but only the mean values are shown $\left({ }^{*} p<0.05\right.$ compared with controls). 


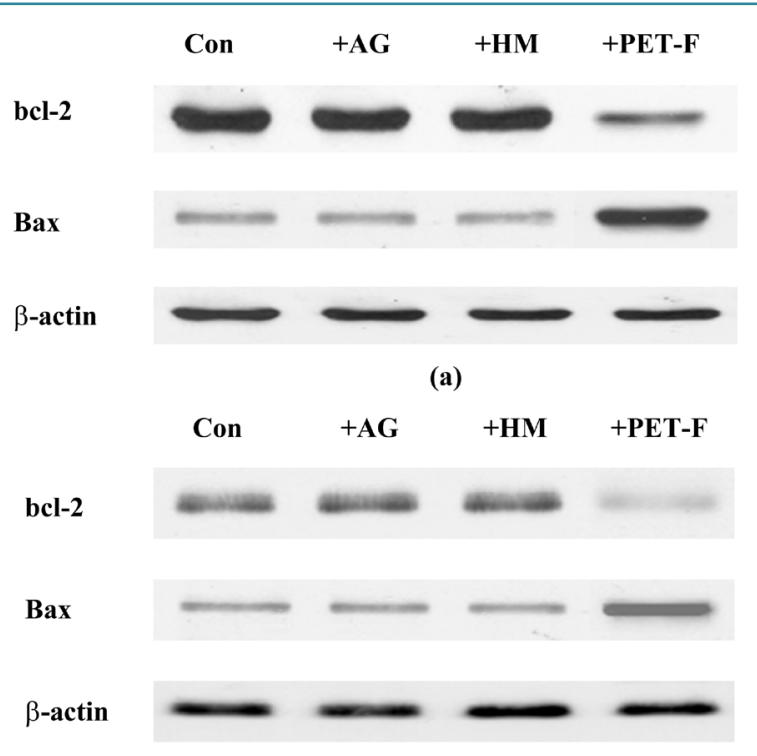

(b)

Figure 3. Western blot analysis on apoptotic regulators. CF33 and CF21 cells were treated with AG, HM, or PET-F at 1000 $\mu \mathrm{g} / \mathrm{ml}$ for $24 \mathrm{~h}$, and they were subjected to Western blot analysis on two apoptotic regulators, bcl-2 and Bax. Autoradiographs of bcl-2 and Bax detected in CF33 (a) and CF21 (b) cells following the indicated treatments (Con, AG, HM, or PET-F) are shown. For a protein loading control, $\beta$-actin was also run and shown here.

cells also showed the similar results of the bcl-2 down-regulation and Bax up-regulation induced by PET-F, but no changes in bcl-2 and Bax expressions were seen with AG or HM (Figure 3(b)). After all, such a significant down-regulation of bcl-2 concomitant with a significant up-regulation of Bax seen in both cancer cells indicates induction of apoptosis because bcl-2 is an anti-apoptotic (growth stimulatory) regulator while Bax is a pro-apoptotic (growth inhibitory) regulator [15]. On the other hand, as AG and HM had little effects on bcl-2 and Bax (compared to controls), the growth reduction induced by these products is more likely attributed to a cytostatic effect, rather than apoptosis. Thus, these findings suggest that only PET-F appears to induce apoptosis, presumably accounting for the superior growth reduction/inhibition.

\subsection{Enhanced Anticancer Effects by Combinations of Various Products and VC}

Since PET-F has been postulated to be activated by VC [16], this possibility was tested together with four other products (AG, HM, AHCC, and YBG). All five products were then tested at the statistically ineffective concentrations of $500 \mu \mathrm{g} / \mathrm{ml}$ and $400 \mu \mathrm{g} / \mathrm{ml}$ in CF33 and CF21 cells, respectively (although AHCC and YBG are ineffective at any concentrations, as shown in Figure 1). Separately, both cancer cells were also treated with the same given concentrations of five products in combination with VC $(200 \mu \mathrm{M})$. After 24-h treatment, cell growth was determined in both cancer cells (Figure 4). Overall, four products (AG, HM, AHCC, and YBG) all failed to show any significant improvements in their anticancer activities with VC in both cancer cells (Figure 4(a) and Figure 4(b)). In contrast, PET-F demonstrated a remarkable potentiation with VC, resulting in nearly complete cell death (>95\%) in these cancer cells (Figure 4(a) and Figure 4(b)). Therefore, only PET-F, not other four products, appears to be exceptionally and superbly potentiated with VC, inducing such severe cell death.

\section{Discussion}

Canine cancers are as prevalent as human cancers and several therapeutic options are currently available; however, the effective and satisfactory treatments are yet required and need to be established timely. In the meantime, as an alternative option for the conventional therapeutic modalities, a number of nutritional supplements 

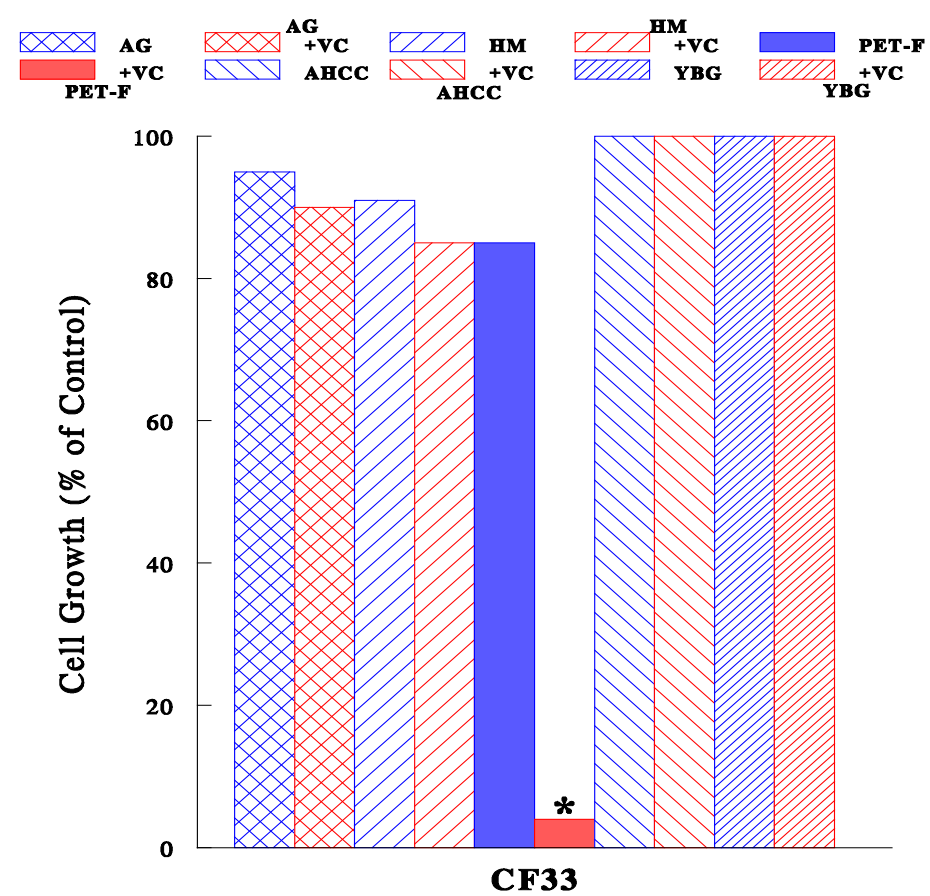

(a)

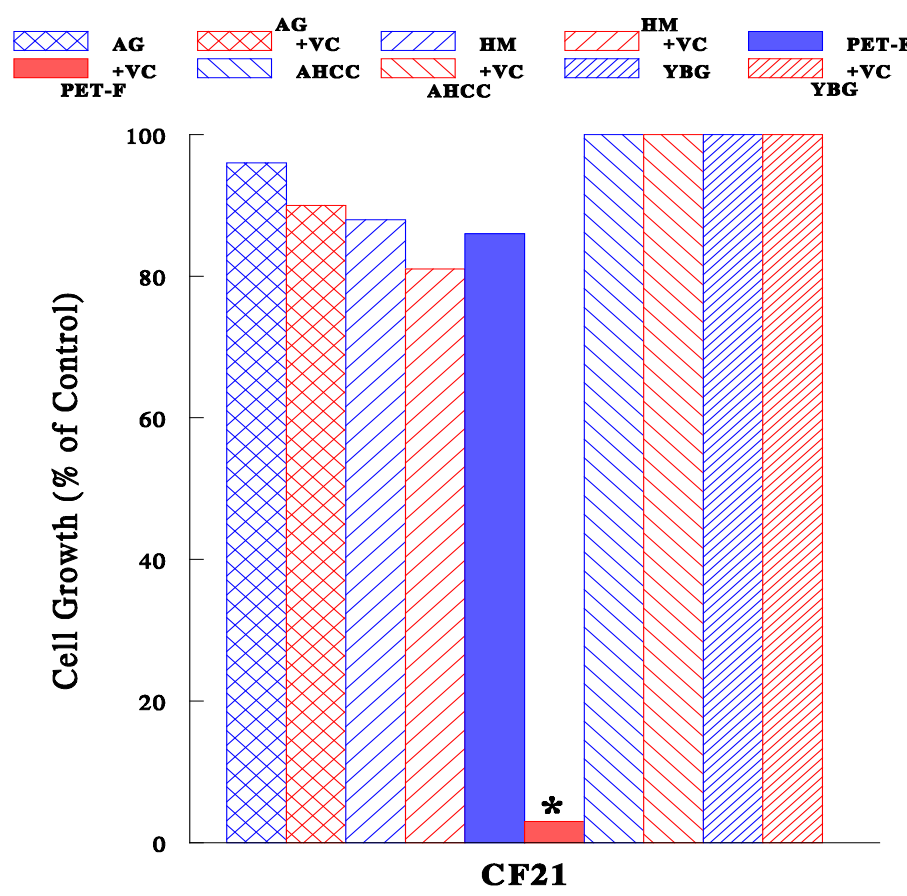

(b)

Figure 4. Combined effects of various products and VC. CF33 and CF21 cells were treated with all five products (AG, HM, PET-F, AHCC, and YBG) at the statistically ineffective concentrations of $500 \mu \mathrm{g} / \mathrm{ml}$ and $400 \mu \mathrm{g} / \mathrm{ml}$, respectively. Separately, both cancer cells were also treated with the same given concentrations of five products in combination with VC $(200 \mu \mathrm{M})$. After $24 \mathrm{~h}$, cell growth of cancer cells were determined and individually plotted in the graphs. The data are mean \pm SD from three separate experiments but only the mean values are shown $(* p<0.01$ compared with cells treated only with PET-F). 
for the (cancer) therapeutic purpose are commercially available and flooded in the market. However, a majority of these supplements/products have not yet been tested or approved for their efficacy on veterinary animals bearing cancers. Hence, our primary objective in the present study was to examine or assess anticancer effects of five selected commercial products on the two types of canine cancer cells, mammary gland cancer (CF33) and soft tissue cancer (CF21) cells.

Among five products tested, PET-F has been confirmed to have a potent anticancer activity as its former formula (DVM Fraction) demonstrated previously [12]. In fact, two other products, AG and HM, also led to a significant growth reduction in CF33 and CF21 cells (despite the high concentrations required), PET-F was more effective than them and was indeed the most potent product. In terms of a relative "susceptibility" (to various products), CF33 cells appear to be less susceptible than CF21 cells. It could be likely due to the different nature of these cancers: canine mammary cancer (CF33) is known to be more aggressive and progressive than soft tissue cancer (CF21) [17] [18], probably accounting for a different susceptibility to various products.

To have an insight into the underlying anticancer mechanism of these three products, we examined their possible effects on cell cycle, which was known to substantially control cell proliferation. Cell cycle analysis showed that the growth reduction induced by PET-F was well correlated with a $\mathrm{G}_{1}$ cell cycle arrest, a blocking of cells entering from the $G_{1}$ to the $S$ phase, resulting in a cell accumulation in the $G_{1}$ phase. This $G_{1}$ arrest has been shown to ultimately lead to the growth inhibition and/or cell death [14]. On the other hand, the growth reduction induced by AG or HM is unlikely associated with the cell cycle regulation but is possibly linked to the other growth regulatory pathway(s) instead. Therefore, a $\mathrm{G}_{1}$ cell cycle arrest induced by PET-F may at least in part account for the resulting growth reduction.

Although the growth reduction induced by three products may follow the different regulatory pathways (cell cycle or unidentified pathways), the same question has been often raised-whether such a growth reduction might be linked to apoptosis. To address this question, we analyzed how two key apoptotic regulators, bcl-2 and Bax, in cancer cells would be affected following the individual treatment of three products. These two regulators play a pivotal role in cell growth in relevant to the apoptotic regulation because bcl-2 is anti-apoptotic while Bax is pro-apoptotic [15]. Hence, balances between two regulators would determine whether cell proliferation will be facilitated or inhibited (leading to apoptosis). The reduced bcl-2 expression with the elevated Bax expression observed in PET-F-treated CF33 and CF21 cells (Figure 3(a) and Figure 3(b)) indicated the growth reduction induced by PET-F was rather associated with apoptosis. Nevertheless, even though both AG and HM were also capable of inducing a significant growth reduction, it was somewhat peculiar to find that such a growth reduction was unlikely attributed to apoptosis. It is thus plausible that the growth reduction induced by AG or HM could be more likely due to a cytostatic effect, rather than apoptosis.

As it has been postulated that " $\beta$-glucan", a major active component of PET-F, could be activated by vitamin C (VC) [16], we examined if anticancer effect of PET-F might be further potentiated with VC. Along with PET-F, four other products were also individually tested with VC. The results showed that four products (AG, $\mathrm{HM}, \mathrm{AHCC}$, and YBG) were little potentiated with VC but PET-F exhibited a prominent potentiation with VC, leading to severe cell death (>95\%) in both CF33 and Cf21 cells. Thus, not all products are the same in terms of their potency and nature, so that only PET-F can be exceptionally potentiated with $\mathrm{VC}$, inducing the profound growth reduction (due to extensive cell death).

After all, PET-F is indeed a promising product with the best anticancer effect on canine cancer cells. However, as it was the in vitro study, it is uncertain whether the effective concentrations ( 400 or $500 \mu \mathrm{g} / \mathrm{ml}$ ) of PET-F could be physiologically achievable or actually effective in vivo. We also need to figure out how such effective concentrations of PET-F in this study would be even translated in the in vivo study. Due to technical difficulties, few studies have yet been performed to determine the attainable physiological concentrations of PET-F in animals. However, PET-F has been studied in dogs bearing intermediate or high-grade lymphomas with an expected survival time of 2 weeks [19]. Such study showed that PET-F was well tolerated in these dogs but no significant improvements or cancer regression was seen with PET-F administration. However, our previous study of DVM Fraction, the former PET-F, showed a significant anticancer or antiproliferative effect on canine lymphoma cells in vitro [12]. This discrepancy could be due to several pathophysiological factors. Particularly, since no physiologically achievable concentration of PET-F has yet been established, it is plausible that the concentration or dosage of PET-F used in this dog study might not be optimal or should have been higher to be effective. Hence, another study using the increasing or several higher doses of PET-F in dogs bearing lymphoma should be performed to see if such higher doses could be more effective. Additionally, it is known and well ac- 
cepted that not all results of the in vitro studies could be comparable to those of the in vivo studies. In this dog (in vivo) study, the pathophysiological conditions of those dogs need to be also considered besides the cancer (lymphoma) status alone. If they were suffering from an aggressive multicentric disease with a progressive metastasis, which have been well documented [1] [20], their vital organs could suddenly start failing, their conditions could be rapidly deteriorating, and they would eventually collapse. It is then hard to presume if there were any ways to just save them or if they were already critical and untreatable because their conditions were too rapidly declining to even have any benefits from PET-F. This may more likely create a discrepancy in the outcomes between the in vitro and the in vivo study. However, it is yet crucial to address if PET-F would be effective on dogs with mammary gland (CF33) or soft tissue (CF21) cancers as it has been shown in this (in vitro) study. More studies are thus required for properly assessing the efficacy of PET-F on various canine cancers.

Lastly, it is also important to address the safety of PET-F for a therapeutic utility. In addition to the above PET-F study on dogs with lymphomas [19], a few toxicity studies of PET-F have also been performed in animals. For instance, a toxicity study using healthy beagle dogs $(\mathrm{n}=10)$ confirmed that no toxicity was seen in any dogs when they received PET-F (3.3 mg/ kg body weight per day) for 10 weeks [21]. Thus, the safety of PET-F appears to be granted but the important task yet remains to establish its physiologically tolerable concentration. Prior to the ultimate PET-F study on dogs with various cancers, we will next focus on the in vivo study of PET-F (as well as some other commercial products) using nude mice (implanted with canine cancer cells) to establish/assess its effective dosage, actual efficacy, and safety.

\section{Conclusion}

In conclusion, the present study shows that three of five commercial products, AG, HM, and PET-F, have a significant anticancer effect on CF33 and CF21 cells, although PET-F has the most potent anticancer activity. Such an anticancer mechanism of PET-F is associated with a $\mathrm{G}_{1}$ cell cycle arrest, which ultimately leads to apoptosis. However, the anticancer mechanism of AG and HM appears to be different from that of PET-F because they have no effects on cell cycle and never induce apoptosis. Their anticancer mechanism should be explored further but it can be assumed at this point that the growth reduction induced by AG and HM is at least due to a cytostatic effect. Moreover, anticancer activity of PET-F, not other four products, can be further potentiated with VC, leading to severe ( $>95 \%)$ cell death in both cancer cells. This PET-F and VC combination could also be another viable therapeutic option. Therefore, PET-F is the most potent and promising product, which can be useful in treating a variety of canine cancers as well as other veterinary cancers.

\section{Acknowledgements}

We thank Mr. Mike Shirota (Mushroom Wisdom, Inc., East Rutherford, NJ) for generously providing us PETFraction $^{\mathrm{TM}}$ in this study.

\section{References}

[1] Richards, K.L. and Suter, S.E. (2015) Man's Best Friend: What Can Pet Dogs Teach Us about Non-Hodgkin's Lymphoma? Immunological Reviews, 263, 173-191. http://dx.doi.org/10.1111/imr.12238

[2] Davis, B.W. and Ostrander, E.A. (2014) Domestic Dogs and Cancer Research: A Breed-Based Genomics Approach. ILAR Journal, 55, 59-68. http://dx.doi.org/10.1093/ilar/ilu017

[3] Mizuno, T. and Zhuang, C. (1995) Maitake, Grifola frondosa: Pharmacological Effects. Food Reviews International, 11, 135-149. http://dx.doi.org/10.1080/87559129509541024

[4] Nanba, H., Hamaguchi, A. and Kuroda, H. (1987) The Chemical Structure of an Antitumor Polysaccharide in Fruit Bodies of Grifola frondosa (Maitake). Chemical and Pharmaceutical Bulletin, 35, 1162-1168.

[5] Kabir, Y., Yamaguchi, M. and Kimura, S. (1987) Effect of Shiitake (Lentinus edodes) and Maitake (Grifola frondosa) Mushrooms on Blood Pressure and Plasma Lipids of Spontaneously Hypertensive Rats. Journal of Nutritional Science and Vitaminology (Tokyo), 33, 341-346.

[6] Adachi, K., Nanba, H., Otsuka, M. and Kuroda, H. (1988) Blood Pressure-Lowering Activity Present in the Fruit Body of Grifola frondosa (Maitake). Chemical and Pharmaceutical Bulletin, 36, 1000-1006.

[7] Kubo, K., Aoki, H. and Nanba, H. (1994) Anti-Diabetic Activity Present in the Fruit Body of Grifola frondosa (Maitake). Biological and Pharmaceutical Bulletin, 17, 1106-1110. 
[8] Kubo, K. and Nanba, H. (1996) The Effect of Maitake Mushroom on Liver and Serum Lipids. Alternative Therapies in Health and Medicine, 2, 62-66.

[9] Nakai, R., Masui, H., Horio, H. and Ohtsuru, M. (1999) Effect of Maitake (Grifola frondosa) Water Extract on Inhibition of Adipocyte Conversion of C3H10T1/2B2C1 Cells. Journal of Nutritional Science and Vitaminology (Tokyo), 45, 385-389.

[10] Gu, C.Q., Li, J.W. and Chao, F.H. (2006) Inhibition of Hepatitis B Virus by D-Fraction from Grifola frondosa: Synergistic Effect of Combination with Interferon- $\alpha$ in HepG2 2.2.15. Antiviral Research, 72, 162-165. http://dx.doi.org/10.1016/j.antiviral.2006.05.011

[11] National Cancer Institute (1992) Developmental Therapeutics Program: In-Vitro Anti-HIV Drug Screening Results. National Science Council F195001, Washington DC.

[12] Konno, S. (2004) Potential Growth Inhibitory Effect of Maitake-D-Fraction on Canine Cancer Cells. Veterinary Therapeutics, 5, 263-271.

[13] Mordente, J.A., Konno, S., Chen, Y., Wu, J.M., Tazaki, H. and Mallouh, C. (1998) The Effects of Brefeldin A (BFA) on Cell Cycle Progression Involving the Modulation of the Retinoblastoma Protein (pRB) in PC-3 Prostate Cancer Cells. Journal of Urology, 159, 275-279. http://dx.doi.org/10.1016/S0022-5347(01)64081-3

[14] Sherr, C.J. (2000) The Pezcoller Lecture: Cancer Cell Cycles Revised. Cancer Research, 60, 3689-3695.

[15] Yip, K.W. and Reed, J.C. (2008) Bcl-2 Family Proteins and Cancer. Oncogene, 27, 6398-6406. http://dx.doi.org/10.1038/onc.2008.307

[16] Morishige, F. (1986) The Role of Vitamin C in Tumor Therapy (Human). In: Meyskens Jr., F.I. and Parasad, K.N., Eds., Vitamins and Cancer: Human Cancer Prevention by Vitamins and Micronutrients, Humana Press, Clifton, 399427. http://dx.doi.org/10.1007/978-1-4612-5006-7_26

[17] Cekanova, M. and Rathore, K. (2014) Animal Models and Therapeutic Molecular Targets of Cancer: Utility and Limitations. Drug Design, Development and Therapy, 8, 1911-1922. http://dx.doi.org/10.2147/DDDT.S49584

[18] Sayama, A., Okado, K., Imaoka, M., Yokouchi, Y., Jindo, T. and Takasaki, W. (2014) Subcutaneous Soft Tissue Sarcoma with Rhabdoid Features in a Dog. Journal of Toxicologic Pathology, 27, 131-138. http://dx.doi.org/10.1293/tox.2013-0044

[19] Griessmayr, P.C., Gauthier, M., Barber, L.G. and Cotter, S.M. (2007) Mushroom-Derived Maitake PET-Fraction as Single Agent for the Treatment of Lymphoma in Dogs. Journal of Veterinary Internal Medicine, 21, 1409-1412. http://dx.doi.org/10.1111/j.1939-1676.2007.tb01967.x

[20] Johnston, S.A., Thamm, D.H. and Legutki, J.B. (2014) The Immunosignature of Canine Lymphoma: Characterization and Diagnostic Application. BMC Cancer, 14, 657-667. http://dx.doi.org/10.1186/1471-2407-14-657

[21] Nippon Zenoaq Kogyo Co. Ltd. (2003) Subacute Toxicity Test of PET-Fraction in Beagle Dogs. Zenoaq Publication, Koriyama, Fukushima. 